\title{
Scale-Independent Aggression: A Fractal Analysis of Four Levels of Human Aggression
}

\author{
Julia J. C. Blau $\mathbb{D}^{1}$ and Alexandra Paxton $\mathbb{D}^{2,3}$ \\ ${ }^{1}$ Department of Psychological Sciences, Central Connecticut State University, New Britain, CT, USA \\ ${ }^{2}$ Department of Psychological Sciences, University of Connecticut, Storrs, CT, USA \\ ${ }^{3}$ Center for the Ecological Study of Perception and Action, University of Connecticut, Storrs, CT, USA \\ Correspondence should be addressed to Julia J. C. Blau; blau@ccsu.edu
}

Received 14 February 2020; Revised 15 September 2020; Accepted 26 October 2020; Published 19 November 2020

Academic Editor: Diego R. Amancio

Copyright (c) 2020 Julia J. C. Blau and Alexandra Paxton. This is an open access article distributed under the Creative Commons Attribution License, which permits unrestricted use, distribution, and reproduction in any medium, provided the original work is properly cited.

\begin{abstract}
Using fractal analyses to study events allows us to capture the scale-independence of those events, that is, no matter at which level we study a phenomenon, we should get roughly the same results because events exhibit similar structure across scales. This is demonstrably true in mathematical fractals but is less assured in behavioral fractals. The current research directly tests the scaleindependence hypothesis in the behavioral domain by exploring the fractal structure of aggression, a social phenomenon comprising events that span temporal scales from minutes of face-to-face arguments to centuries of international armed conflicts. Using publicly available data, we examined the temporal fractal structure of four scales of aggression: wars (very macrolevel, worldwide data), riots (macrolevel, worldwide data), violent crimes (microlevel, data gathered from cities and towns in the United States of America), and body movement during arguments (very microlevel, data gathered on American participants). Our results lend mixed support to the scale-independence hypothesis and provide insight into the self-organization of human interactions.
\end{abstract}

\section{Introduction}

Some phenomena are fig simply not suited for typical summary statistics such as means and standard deviations [1]. From neurons [2] to nebulae [3] and geology [4] to geography [5], many phenomena exhibit fractality, a phenomenon where patterns recur at all spatiotemporal scales. Fractals generated by purely mathematical processes [6] exhibit perfect self-similarity, with patterns that recur identically at all possible levels of observation. However, natural fractals (e.g., coastlines, clouds, and branching of the lungs; for review, see $[7,8]$ ) have only rough self-similarity, in other words, having patterns that recur in similar but not identical ways across nearly all possible levels of observation.

The perfect self-similarity of mathematical fractals and the rough self-similarity of natural fractals have led to the scale-independence hypothesis. Assuming a phenomenon is fractal, analysis of a single spatial or temporal scale will yield insights that apply equally to the entire fractal phenomenon because fractals exhibit similar or identical structure across scales $[9,10]$. This is empirically demonstrable for mathematical fractals, but it is-to date-less assured for behavioral fractals. While fractal analyses have been performed on different types of events at different scales, (e.g., [11-14]), no single study - to our knowledge-has explored a single type of event across all feasible temporal scales of human activity.

The current research aims to fill this gap by exploring the fractal structure of aggression, a social phenomenon comprising events that span temporal scales from minutes of face-to-face arguments to centuries of international armed conflicts. Although any number of other social contexts should also exhibit fractal structure, we chose aggression because it is a measurable social phenomenon with powerful real-world consequences that are more likely to be recorded than events in other contexts (e.g., friendships and romantic relationships). We examined the temporal fractal structure 
of four scales of aggression: wars (very macrolevel), riots (macrolevel), violent crimes (microlevel), and body movement during arguments (very microlevel).

\section{Method}

2.1. Corpus. For this study, we created a corpus of heterogenous data tracking acts of aggression across four temporal scales. The data were combined from a variety of existing sources; we describe each in greater detail below (see Figure 1).

2.1.1. Very Macrolevel: Wars. We attempted to gather a complete list of all recorded wars. Our operational definition of war is organized and sustained armed conflict between different countries or different factions within a country (i.e., civil war). We derived our data from Wikipedia (https://en. wikipedia.org/wiki/Category:Lists_of_wars_by_date), which has been shown in empirical and qualitative work to be accurate, reliable, and comprehensive in areas from political science to psychopharmacology [15-19] (however, for a conflicting perspective on the completeness of drug information on Wikipedia, see [20]). Wikipedia provided the most comprehensive list we could find, with a record of 2,504 wars starting between c. 3100 BCE and 2019 CE. From this, we derived a time series of interwar intervals from war onset to war onset. When multiple wars began in the same year, we divided the year evenly between them. We ignored both the length and location of the wars. It is worth mentioning that there is a bias in terms of the location of recorded wars, particularly early on the list. For example, the first time that either modern-day North or South America appears on the list is in $537 \mathrm{CE}$, over 300 wars into the list. Presumably, other wars occurred on the North and South American continents during that time, but records of those wars were not included in our source's list. Having said that, even when the list is constrained to the worldwide records of the relatively modern era (i.e., after $1000 \mathrm{CE}$ ), the results are the same. The interval scale is years.

2.1.2. Macrolevel: Riots. We attempted to gather a complete list of all recorded riots. Our operational definition of a riot is a spontaneous violent uprising by a crowd. The boundary between a riot and a war is sometimes a blurry one, and on at least one occasion, a riot became a war (i.e., the 100-hour Soccer Wars between Honduras and El Salvador in July 1969 [21]). Once again, Wikipedia's list was the most comprehensive (https://en.wikipedia.org/wiki/List_of_riots); however, we eliminated the first five because exact dates could not be found, leaving us with a list of 757 events starting between $1650 \mathrm{CE}$ and 2019 CE. From this, we derived a time series of inter-riot intervals from riot onset to riot onset. We ignored the length and location of the riots. The interval scale is days.

2.1.3. Microlevel: Violent Crimes. Our goal was to gather violent crime data from at least one city or town in every state in the United States of America. Unfortunately, we were unable to do so, as crime statistics were not available for every state. Our operational definition for violent crime was person-to-person aggression with the intent to cause harm. States across the USA categorize crimes differently, so we utilized a list of heuristics (see Appendix A). We gathered data from 42 states using a variety of the most reliable methods available to us (e.g., police blotters, government reporting sites, and Freedom of Information Act requests). For each city or town, we gathered a minimum of 512 data points, with a goal of 1,000 data points (range: 517-3,582 crimes per town [22]) (for some towns, records did not extend backwards in time far enough to allow for the higher end; for others, ample available data were provided. Rather than truncate the series, we used all available data). The datasets for each city spanned real-time windows of 9-4,109 days, starting as early as Jan. 3, 2008, and ending as late as Apr. 14, 2019. From this, we derived time series of intercrime-intervals from crime onset to crime onset. The interval scale is minutes.

Data were also collected on a number of other cities but were removed from analysis for the following reasons: (1) Chicago (IL), removed because the reporting timescale (minutes) was too coarse-grained for the density of the data (cf. a more appropriate timescale like seconds); (2) LaGrange (GA), removed because their "violent crime" categories included crimes that were outside of the bounds of our heuristics; (3) Wilmore (KY), removed because all crime was reported as occurring at midnight; (4) Lakewood (WA), removed because all crime was reported as occurring at midnight; (5) Seattle (WA), removed because of unexplained time gaps in the data downloaded from the portal; (6) Albuquerque $(\mathrm{AZ})$, removed due to reporting errors in the data downloaded from the portal; and (7) Louisville (KY), removed because crime labels were insufficiently clear to delineate between violent and nonviolent crime.

2.1.4. Very Microlevel: Arguments. Our smallest unit of analysis focused on a reanalysis of publicly available movement data from face-to-face conversations about personally held conflicting opinions [23]. Briefly, these data were collected on two interacting participants (largely strangers) who held two 8-minute conversations: an affiliative conversation about a topic of joint interest (e.g., favorite entertainment media) and an argument about a topic of joint disagreement identified by the experimenter via both participants' individual survey responses (e.g., abortion and death penalty). During each conversation, participants wore head-mounted accelerometers from Google Glass (Google, Inc.;https:/google.com/glass) running PsyGlass opensource experimental software [24] (for more detailsincluding additional experimental conditions-see [25]). From the continuous movement data from each individual, we identified movement events as accelerometer activity over the 90th percentile of movement activity (specific to each conversation of each individual). We then derived a time series of inter-movement-intervals from movement onset to movement onset, for each participant in each condition ( $n=21$ dyads, 42 conversations, and 84 total time series). The interval scale is seconds. 

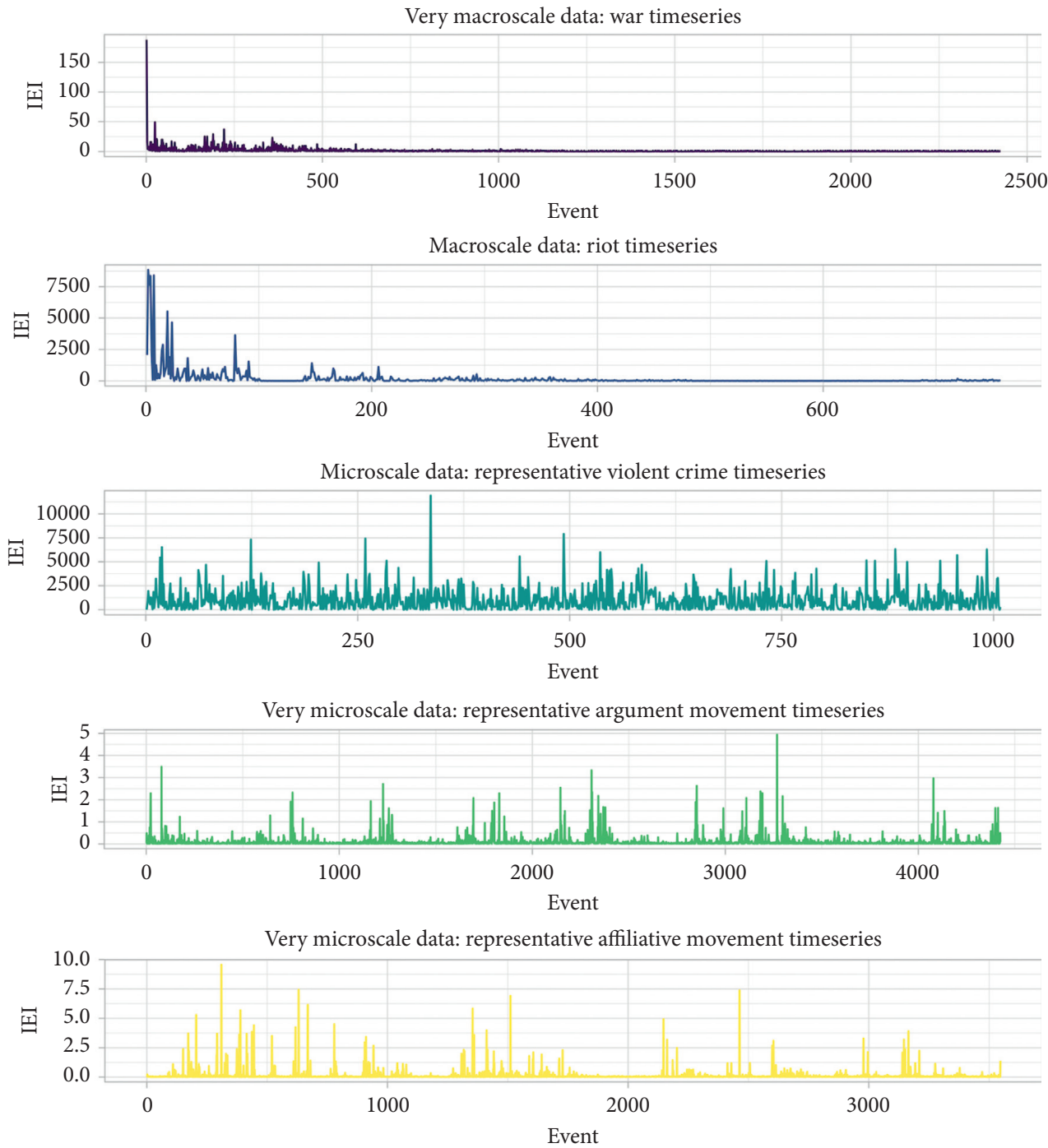

FIgURE 1: Complete time series for Wars and Riots, representative time series for Crime (Applewood, WI) and Argumentative/Affiliative Conversations. Crime and Conversation samples displayed are closest to the group mean $\mathrm{H}$.

2.2. Analysis. While there are a multitude of ways to measure fractality [26], we chose to analyze all resulting time series with detrended fluctuation analysis (DFA [27]), a conservative method that is robust to changes in series length and distribution ([28], but see [29]). DFA consists of five steps. First, it removes overall trends which can overwhelm the analysis. Second, it divides the time series into successively smaller bins, calculating the best fit line in each bin at each bin size. Third, it calculates the average root mean square (ARMS, a measure of the average error of the best fit line) for each bin size. Fourth, it plots $\log$ (ARMS) by $\log$ (bin size). Fifth, it calculates the linear regression of that plot; the slope of this line is the Hurst exponent $(H)$ for step-by-step equations, (see [27] see Figure 2). $H$ is a measure of the structure of a time series. If $0.5<H<1.0$, then the time series is fractal. The 0.5 end of the spectrum is more random; the 1.0 end exhibits more order. Moreover, differences within this range have been shown to have consequences for cognition, emotion, and memory (e.g., $[11,30])$. Because of the size of the numbers involved, differences in $H$ as small as 0.1 are considered significant, but anything smaller than that is usually not.

2.3. Materials, Data, and Code Availability. All analyses were based on publicly available data. All code and data (including derived data; e.g., city-level Hurst exponents, additional plots of time series, and fluctuation by bin size plots) are openly available at https://osf.io/8qcya/.

\section{Results and Discussion}

3.1. Scale Independence. If the scale-independence hypothesis is supported, we would expect a difference of less than 0.1 between the different levels of aggression in terms of their $H$. As expected, Wars $\left(H_{\text {war }}=0.743\right)$, Riots $\left(H_{\text {riot }}=0.741\right)$, and Arguments $\left(H_{\text {argument }}=0.722, S D=0.134\right)$ are all roughly similar. Moreover, neither Wars nor Riots differ 


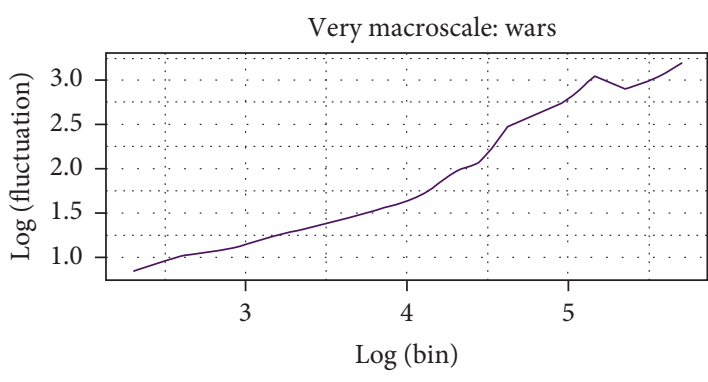

Microscale: riots representative municipality

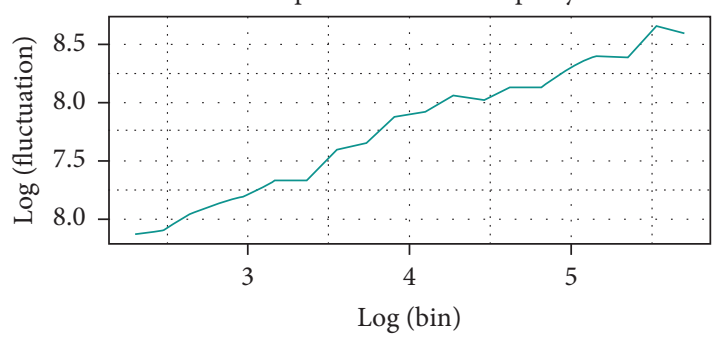

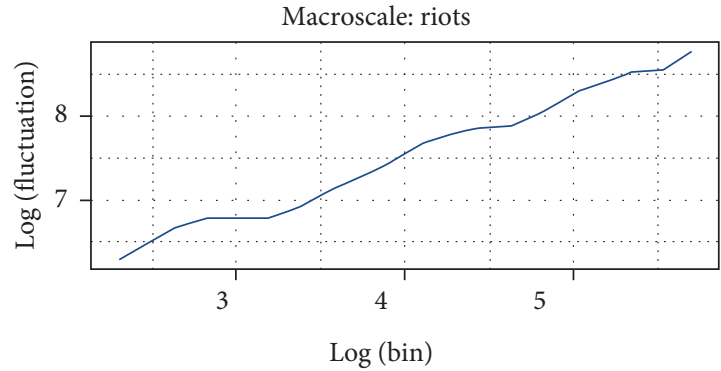

Very microscale: representative affiliative

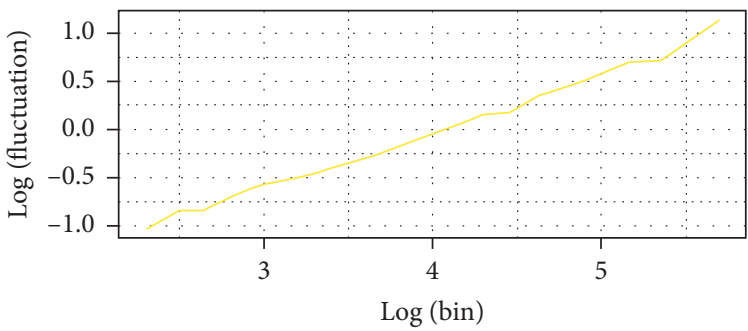

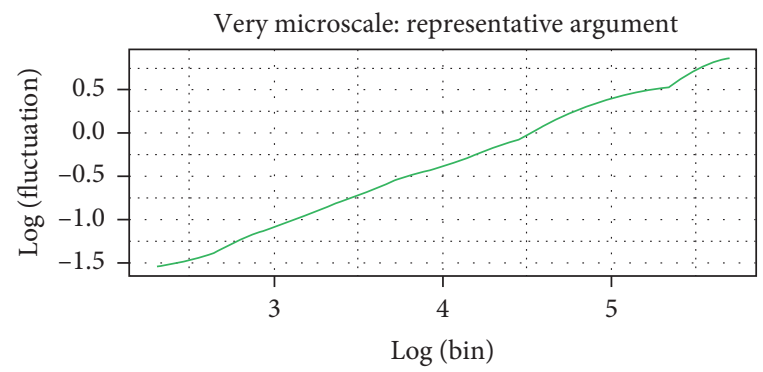

Figure 2: $\log ($ fluctuation) by $\log$ (bin size) plots for Wars and Riots; representative $\log ($ fluctuation by $\log ($ bin size) plots for Crime (Applewood, WI), and Argumentative/Affiliative Conversations. Crime and Conversation samples displayed are closest to the group mean $\mathrm{H}$.

from Arguments in two single-sample two-tailed $t$-tests, $t(83)=1.44, p=0.15$ and $t(83)=1.30, p=0.20$ (respectively). However, Violent Crimes (mean $H_{\text {crime }}=0.534$, $S D=0.12$ ) were significantly different from all three other levels in a series of single-sample two-tailed $t$-tests (vs. Wars: $t[41]=9.94, p<0.0001$; vs. Riots: $t[41]=9.84, p<0.0001$; vs. Arguments: $t[124]=7.16, p<0.0001$, see Figure 3).

For the arguments, we were also able to compare very microlevel aggression events to a similar event of a different affective character (i.e., affiliative instead of argumentative). For the scale-independent hypothesis to hold, it has to be the case that concordance of fractal structure is more than coincidental (that is, if all conversations-not just those of an aggressive nature-exhibit the same level of fractality, which would trivialize the concordance). As expected, affiliative conversations were significantly lower on the fractal spectrum $\left(H_{\text {affiliative }}=0.637 ; S D=0.077\right)$ than Arguments, $t(82)=-3.57, p=0.001$ (two-tailed).

Taken together, our results find mixed support for the scale-independence hypothesis. The Wars, Riots, and Arguments data support the scale-independence hypothesis, but we do not see a similar fractal structure in the Violent Crime data.

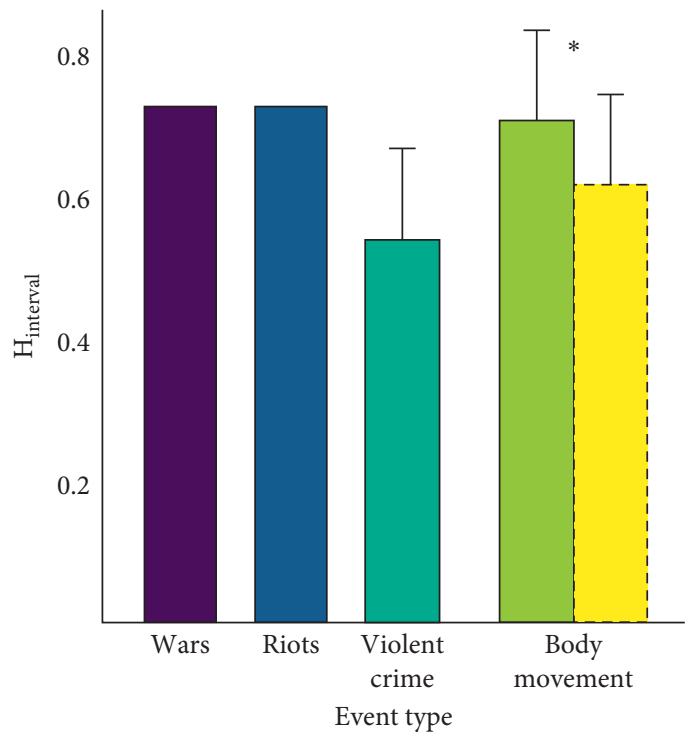

Figure 3: Scale-Independence of Aggression. $(H)_{\text {interval }}$ for the four levels of aggression (solid line bars). $(H)_{\text {argument }}$ (green bar, solid line) was in concordance with $(H)$ wars and $(H)$ riots, but significantly different from $(H)_{\text {affiliate }}$ (yellow bar, dotted line). 


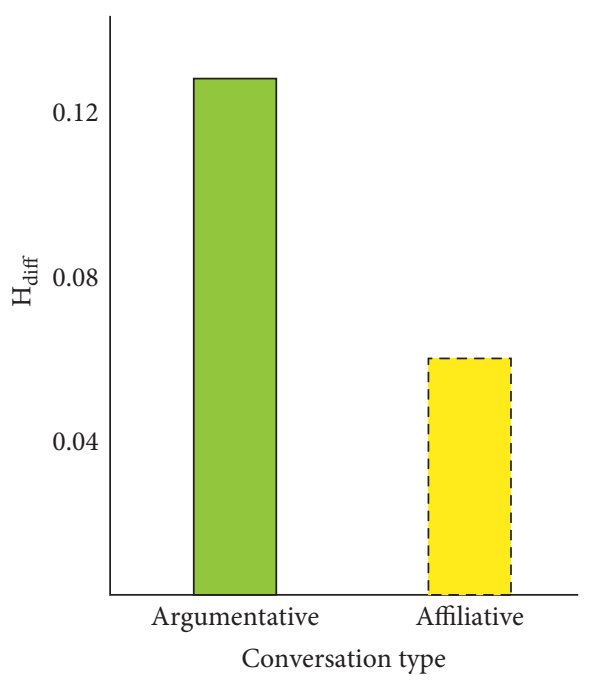

Figure 4: Dynamics of Interaction Type. Dyads having an argumentative conversation (green bar, solid line) were significantly farther apart in terms of the fractal structure of their movement than dyads having an affiliative conversation (yellow bar, dotted line).

3.2. Limitations and Suggestions for Further Research. While analyses of three of the four scales targeted here support the scale-independence hypothesis, the Violent Crime data were notably different from the others. There are several not-mutually-exclusive possibilities for this. First, violent crime is notoriously underreported [31], especially in the United States [32]; assuming which crimes are reported is unsystematic with respect to time (although they may, of course, be systematically underreported due to other factors, such as crime type or location), this might result in the time series appearing to be more random. One way to address this possibility would be to compare the fractality of violent crime across different countries where the so-called "dark figure of crime" is of varying levels. While such underreporting is notoriously difficult to estimate [33, 34], comparisons across different municipalities may provide insights into the impact of underreporting on fractality.

Second, as mentioned above, the sources for the violent crime statistics were of varying quality; it is entirely possible that low fidelity reporting resulted in skewed results. Much like the first possibility, this could be investigated by comparing the fractality of Violent Crime across localities with different reporting qualities.

Finally, it may be that the scale-independence hypothesis simply does not hold at this level of analysis. However, given previous research has shown that many different aspects of city life exhibit power-law scaling [35] (for more on the connection between fractals and power-law scaling, see [36]), we find this to be the least compelling possibility.

While the current work is able to look across scales of human activity or behavior, we here limit our scope to a single kind or character of human behavior. We therefore find ourselves attempting, for the most part, to confirm a null result, that is, identifying that there is no difference among the fractal nature of these scales of a particular activity. Future work could, then, examine other kinds or characters of human behavior in order to provide comparisons both within and across scales but also across behaviors. Some creativity may be needed to identify types of behavior or activities that are as well-documented across temporal scales as aggression is, but we think that identifying activities that are focused on creation or affiliation may be a particularly compelling next step.

Importantly, although the present work provides some evidence of the scale-independence of aggression in humans, we are unable to provide an explanation for these dynamics. We build on a long list of related work in political science that has demonstrated fractality in other dimensions of international conflict, (e.g., [37-40]), and connect it to other behavioral research at interpersonal scales. In doing so, we see our work as one small contribution toward a more unified theory of scale-free self-organizing dynamics that encompasses human behavior at the individual scale, (e.g., [41]), and beyond.

3.3. Complexity Matching in Movement. In addition to providing mixed evidence for the scale-independence hypothesis, our study also builds on previous work on complexity matching during interaction, (e.g., [42]). Not only did affiliative and argumentative conversations differ by overall $H$, participants' fractality coupled differently in each conversational context. For each pair, we calculated $\mathrm{H}_{\text {diff }}=\left|\mathrm{H}_{\text {participant } 1}-\mathrm{H}_{\text {participant } 2}\right| . \quad H_{\text {diff }}$ was significantly higher in the argumentative condition $(M=0.133$, $S D=0.135)$ than in the affiliative condition $(M=0.06$, $S D=0.057), t(40)=-2.10, p=0.042$, two tails (see Figure 4). In other words, argument disrupts the fractal coupling of movement, as also seen in moment-to-moment coupling of movement [25] and fractal coupling of speech [42].

\section{Conclusion}

The scale-independent hypothesis has been an implicit assumption undergirding research on the fractality of human behavior. We here provide mixed empirical evidence for 
scale-independence in the temporal dynamics of human behavior by exploring the temporal structure of acts of aggression, uncovering evidence of scale-independence across very macro (wars), macro (riot), and very micro (arguments) scales but not micro (violent crime) scales. Our findings support the validity of using fractal analyses on human behavior to understand these phenomena across multiple scales; however, we raise important questions about the impact of data quality on such analyses. Although the current work examines only one type of human behavior-aggression-future work should target other kinds and characters of human activities that are well-documented across temporal or spatial scales to continue testing the scale-independence hypothesis in human dynamics.

\section{Appendix}

\section{A. Violent Crime Heuristics}

We defined violent (or aggressive) crime as any crime that is person on person with the intent to cause harm, whether that happened in person or over the Internet. As we collected data, we kept a list of borderline cases for consistency.

\section{Excluded}

Arson-was presumed to be committed with the intent to damage property, not a person;

Burglary-entry without force (intent to commit)- excluded because only intent to commit burglary; Child abandonment;

Crimes listed as "other" without any other information;

Disturbing the peace (unless includes fighting);

Driving to endanger;

Endangering the welfare of a child - child abuse is different;

Entering unlawfully, but no intimidation of owners; For multiple offenses happening in the same incident, count it only once;

Harassment by telephone;

Hit and run misdemeanors (meaning hitting property but not a person);

Interference with a public officer (unless specifies includes violence);

Kidnapping (unless it specifies violence);

Other Sex Crimes (not commercialized) - encompasses indecent exposure/sexual harassment, not necessarily person-on-person violence;

Property damage (ex. beating up someone's car);

Theft with no violent intent (theft with violent intent is called burglary);

Unlawful possession of a weapon.

"Weapons Violations" - in data set provided by the Philadelphia Police Department which also included data points such as:

(i) "Aggravated Assault Firearm" (ii) "Other Assault"

(iii) "Assault Firearm"

Therefore, it is thought that "weapons violations" in this case included incidents of unlawful weapon possessions, not necessarily person-on-person violence due to data set's distinctions.

Included

Adult and juvenile crime;

Animal abuse.

Any classification that had the parent classification of "Assault", "Robbery", or "Breaking and Entering" (Which denotes burglary).

This includes more specific and unusual categories such as:

(i) "Stalking Mal"

(ii) "Stalking"

(iii) "UNF Incident"

(iv) "Warr Arr Fel"

(v) "Tamper Witness"

Child Abuse

Criminal threatening/domestic violence terrorizing

Disorderly Conduct/Fighting/Reckless Conduct

Domestic Abuse

Hit and run felony (meaning hit a person, not just property)

Human trafficking

Road rage-although the data set did not always specify the outcome

Sexual Assault

Detroit, Michigan police use different classifications for criminal sexual conduct. What was included:

(i) "CSC $1^{\text {st }}$ Degree" - denotes violent rape in Michigan law, including oral, anal, and vaginal rape, and rape by object. This criminal sexual charge includes penetration of any body cavity of the victim, which differs from lower criminal sexual charges.

(ii) "CSC $2^{\mathrm{ND}}$ Degree" - denotes a sexual altercation that involves a victim that is under the perpetrator's authority or care, and involves an altercation of violence resulting in direct bodily harm to the victim.

What was excluded:

(i) "CSC $4{ }^{\mathrm{TH}}$ Degree-Forcible Contact" - is treated in Michigan as a misdemeanor, without an onus on a violent intent legally, and does not involve a victim that has been taken advantage of by a person in authority or their care giver. This charge does not include bodily harm that can be included in the heuristics of person-to-person violence. 
(ii) "CSC $3^{\mathrm{RD}}$ Degree" - denotes a sexual altercation involving a victim underneath the perpetrator's authority or care, and does not involve violent bodily harm to the victim. In other words, this differs from CSC $2^{\text {nd }}$ degree because this cannot include direct bodily harm that could be included in the heuristics established as person-to-person violence. This charge is, simply put, a charge made explicitly for coercion cases.

\section{Data Availability}

All data in this paper were publicly available datasets.

\section{Conflicts of Interest}

The authors declare that they have no conflicts of interest.

\section{Acknowledgments}

The authors would like to thank Isabella Cipollone (UConn) for assistance with data entry for riots, as well as Amy Ferrante (CCSU) and the undergraduate research team in the Blau lab for their assistance mining violent crime data. A previous version of this work was presented at the International Conference of Perception and Action, 2019.

\section{References}

[1] L. S. Liebovitch, Fractals and Chaos Simplified for the Life Sciences, Oxford University Press, New York, NY, USA, 1998.

[2] G. F. Zebende, F. M. Oliveira Filho, and J. A. Leyva Cruz, "Auto-correlation in the motor/imaginary human EEG signals: a vision about the FDFA fluctuations," PloS One, vol. 12, no. 9, Article ID e0183121, 2017.

[3] M. Joyce, P. W. Anderson, M. Montuori, L. Pietronero, and F. S. Labini, "Fractal cosmology in an open universe," Europhysics Letters (EPL), vol. 50, no. 3, pp. 416-422, 2000.

[4] W. Lin, X. Li, Z. Yang, L. Lin et al., "A new improved threshold segmentation method for scanning images of reservoir rocks considering pore fractal characteristics," Fractals, vol. 26, no. 02, Article ID 1840003, 2018.

[5] J. Xiao and Z.-g. Xia, "Fractals in physical geography," Progress in Physical Geography: Earth and Environment, vol. 20, no. 2, pp. 178-191, 1996.

[6] B. B. Mandelbrot, The fractal geometry of nature, W. H. Freeman, New York, NY, USA, 1983.

[7] M. Newman, "Power laws, Pareto distributions and zipf's law," Contemporary Physics, vol. 46, no. 5, pp. 323-351, 2005.

[8] C. T. Kello and G. C. Van Orden, "Soft-assembly of sensorimotor function," Nonlinear Dynamics Psychology Life Sciences, vol. 13, no. 1, pp. 57-78, 2009.

[9] B. J. West and M. Shlesinger, "The noise in natural phenomena," American Scientist, vol. 78, no. 1, pp. 40-45, 1990.

[10] N. Bez and S. Bertrand, "The duality of fractals: roughness and self-similarity," Theoretical Ecology, vol. 4, no. 3, pp. 371-383, 2011.

[11] J. J. C. Blau, S. C. Petrusz, and C. Carello, "Fractal structure of event segmentation: lessons from reel and real events," Ecological Psychology, vol. 25, no. 1, pp. 81-101, 2013.

[12] N. Chater and G. D. A. Brown, "Scale-invariance as a unifying psychological principle," Cognition, vol. 69, no. 3, pp. B17-B24, 1999.
[13] T. J. Davis, T. R. Brooks, and J. A. Dixon, "Multi-scale interactions in interpersonal coordination," Journal of Sport and Health Science, vol. 5, no. 1, pp. 25-34, 2016.

[14] J. Szary, R. Dale, C. T. Kello, and T. Rhodes, "Patterns of interaction-dominant dynamics in individual versus collaborative memory foraging," Cognitive Processing, vol. 16, no. 4, pp. 389-399, 2015.

[15] J. M. Heilman, E. Kemmann, M. Bonert et al., "Wikipedia: a key tool for global public health promotion," Journal of Medical Internet Research, vol. 13, no. 1, p. e14, 2011.

[16] A. R. Thomas, "Wikipedia as a data source for political scientists: accuracy and completeness of coverage," PS: Political Science \& Politics, vol. 44, no. 02, pp. 339-343, 2011.

[17] J. Giles, "Internet encyclopaedias go head to head," Nature, vol. 438, no. 7070, pp. 900-901, 2005.

[18] J. Kräenbring, T. M. Penza, J. Gutmann et al., “Accuracy and completeness of drug information in Wikipedia: a comparison with standard textbooks of pharmacology," PloS One, vol. 9, no. 9, Article ID e106930, 2014.

[19] N. J. Reavley, A. J. Mackinnon, A. J. Morgan et al., "Quality of information sources about mental disorders: a comparison of wikipedia with centrally controlled web and printed sources," Psychological Medicine, vol. 42, no. 8, pp. 1753-1762, 2012.

[20] K. A. Yap, H. H. Polen, M. N. K. Boulos, and J. H. Dzenowagis, "Scope, completeness, and accuracy of drug information in wikipedia," Annals of Pharmacotherapy, vol. 42, no. 12, pp. 1814-1821, 2008.

[21] T. Wallace, "The soccer wars: hispanic immigrants in conflict and adaptation at the soccer borderzone," Napa Bulletin, vol. 31, no. 1, pp. 64-77, 2009.

[22] R. Weron, "Estimating long-range dependence: finite sample properties and confidence intervals," Physica A: Statistical Mechanics and Its Applications, vol. 312, no. 1-2, pp. 285-299, 2002.

[23] A. Paxton and R. Dale, "Dual conversation constraints: data and code for "interpersonal movement synchrony responds to high- and low-level conversational constraints,", 2017.

[24] A. Paxton, K. Rodriguez, and R. Dale, "PsyGlass: capitalizing on google glass for naturalistic data collection," Behavior Research Methods, vol. 47, no. 3, pp. 608-619, 2015.

[25] A. Paxton and R. Dale, "Interpersonal movement synchrony responds to high- and low-level conversational constraints," Frontiers in Psychology, vol. 8, p. 1135, 2017.

[26] R. Lopes and N. Betrouni, "Fractal and multifractal analysis: a review," Medical Image Analysis, vol. 13, no. 4, pp. 634-649, 2009.

[27] C.-K. Peng, S. V. Buldyrev, S. Havlin, M. Simons, H. E. Stanley, and A. L. Goldberger, "Mosaic organization of DNA nucleotides," Physical review e, vol. 49, no. 2, pp. 1685-1689, 1994.

[28] M. J. Cannon, D. B. Percival, D. C. Caccia, G. M. Raymond, and J. B. Bassingthwaighte, "Evaluating scaled windowed variance methods for estimating the hurst coefficient of time series," Physica A: Statistical Mechanics and Its Applications, vol. 241, no. 3-4, pp. 606-626, 1997.

[29] R. M. Bryce and K. B. Sprague, "Revisiting detrended fluctuation analysis," Scientific Reports, vol. 2, p. 315, 2012.

[30] J. M. Gordon, J. J. C. Blau, and Carello, "The effect of fractal event structure on anxiety and memory," in Studies in Perception and Action, E. Charles and L. J. Smart, Eds., pp. 22-26, Taylor \& Francis, New York, NY, USA, 2011.

[31] H. E. Williams, S. W. Bowman, and J. T. Jung, "The limitations of government databases for analyzing fatal officer-involved 
shootings in the United States," Criminal Justice Policy Review, vol. 30, no. 2, pp. 201-222, 2019.

[32] S. L. Myers, "Why are crimes underreported? What is the crime rate? Does it "really" matter?" Social Science Quarterly, vol. 61 , no. 1, pp. 23-43, 1980.

[33] L. A. J. Quetelet, "Sur l'homme et le développement de ses facultés, ou Essai de physique sociale," 1869.

[34] F. S. Pezzella, M. D. Fetzer, and T. Keller, "The dark figure of hate crime underreporting," American Behavioral Scientist, Article ID 0002764218823844, 2019.

[35] L. M. A. Bettencourt, J. Lobo, D. Helbing, C. Kühnert, and G. B. West, "Growth, innovation, scaling, and the pace of life in cities," Proceedings of the National Academy of Sciences, vol. 104, no. 17, pp. 7301-7306, 2007.

[36] J. H. Brown, V. K. Gupta, B.-L. Li, B. T. Milne, C. Restrepo, and G. B. West, "The fractal nature of nature: power laws, ecological complexity and biodiversity," Philosophical Transactions of the Royal Society of London. Series B: Biological Sciences, vol. 357, no. 1421, pp. 619-626, 2002.

[37] J. C. Bohorquez, S. Gourley, A. R. Dixon, M. Spagat, and N. F. Johnson, "Common ecology quantifies human insurgency," Nature, vol. 462, no. 7275, pp. 911-914, 2009.

[38] L.-E. Cederman, "Modeling the size of wars: from billiard balls to sandpiles," American Political Science Review, vol. 97, no. 01, pp. 135-150, 2003.

[39] A. Clauset, M. Young, and K. S. Gleditsch, "On the frequency of severe terrorist events," Journal of Conflict Resolution, vol. 51, no. 1, pp. 58-87, 2007.

[40] L. F. Richardson, "Variation of the frequency of fatal quarrels with magnitude," Journal of the American Statistical Association, vol. 43, no. 244, pp. 523-546, 1948.

[41] E. A. F. Ihlen and B. Vereijken, "Interaction-dominant dynamics in human cognition: Beyond $1 / f \alpha$ fluctuation," Journal of Experimental Psychology: General, vol. 139, no. 3, pp. 436-463, 2010.

[42] D. H. Abney, A. Paxton, R. Dale, and C. T. Kello, "Complexity matching in dyadic conversation," Journal of Experimental Psychology: General, vol. 143, no. 6, pp. 2304-2315, 2014. 